DOI 10.4171/JEMS/203

Bernhard Ruf · P. N. Srikanth

\title{
Singularly perturbed elliptic equations with solutions concentrating on a 1-dimensional orbit
}

Received June 7, 2007 and in revised form April 2, 2008

\begin{abstract}
We consider a singularly perturbed elliptic equation with superlinear nonlinearity on an annulus in $\mathbb{R}^{4}$, and look for solutions which are invariant under a fixed point free 1-parameter group action. We show that this problem can be reduced to a nonhomogeneous equation on a related annulus in dimension 3. The ground state solutions of this equation are single peak solutions which concentrate near the inner boundary. Transforming back, these solutions produce a family of solutions which concentrate along the orbit of the group action near the inner boundary of the domain.
\end{abstract}

\section{Introduction}

We consider the following superlinear elliptic boundary value problem on the annulus $A=\left\{x \in \mathbb{R}^{4}|0<a<| x \mid<b\right\}$ :

$$
\begin{cases}-\varepsilon^{2} \Delta u+u=u^{p} & \text { in } A, \\ u(x)>0, & \text { in } A, \\ u(x)=0, & \text { on } \partial A .\end{cases}
$$

Here $p>1$, and $\varepsilon^{2}$ is a singular perturbation parameter.

In the pioneering papers [10-13] qualitative properties of the least energy solution for this singularly perturbed equation (with varying boundary conditions) have been studied. In particular, W.-M. Ni and J. Wei showed in [13] that the least energy solutions of equations of form (1) concentrate, for $\varepsilon \rightarrow 0$, to single peak solutions, whose maximum points $x_{\epsilon}$ converge to a point $x_{0}$ with maximal distance from the boundary $\partial \Omega$. Furthermore, $\mathrm{Ni}$ and Wei gave precise decay estimates for these solutions.

Another type of concentrating solutions was studied by A. Ambrosetti, A. Malchiodi and W.-M. Ni in [1] (see also [5]); they consider solutions which concentrate on spheres, i.e. on $N-1$-dimensional manifolds. Such solutions are of particular interest for applica-

B. Ruf: Dipartimento di Matematica, Università degli Studi di Milano, Via Saldini, 50, 20123 Milano, Italy; e-mail: ruf@mat.unimi.it

P. N. Srikanth: TIFR Centre for Applicable Mathematics, Bangalore 560 065, India; e-mail: srikanth@math.tifrbng.res.in 
tions of the equation to models of activator-inhibitor systems in biology (see the survey [14]). In their paper, Ambrosetti, Malchiodi and Ni consider equation (1) in the presence of a potential $V(r)$, i.e.

$$
\begin{cases}-\varepsilon^{2} \Delta u+V(|x|) u=u^{p} & \text { in } A \subset \mathbb{R}^{N} \\ u(x)=0 & \text { on } \partial A .\end{cases}
$$

They showed that if the function $M(r)=r^{n-1} V^{\theta}(r)$ with $\theta=(p+1) /(p-1)-1 / 2$ satisfies $M^{\prime}(b)<0$ (resp. $\left.M^{\prime}(a)>0\right)$, then there exists a family of radial solutions which concentrate on $|x|=r_{\varepsilon}$ with $r_{\varepsilon} \rightarrow b$ (resp. $r_{\varepsilon} \rightarrow a$ ) as $\varepsilon \rightarrow 0$.

It has been conjectured that for $N \geq 3$ there could also exist solutions concentrating on some manifolds of dimension $\mathrm{k}$ with $1 \leq k \leq N-2$.

In this paper we will prove

Theorem 1. Let $1<p<5$. Then for problem (1) there exists a family of positive solutions which concentrate on a 1-dimensional orbit $T_{\tau} x_{\varepsilon}, \tau \in[0,2 \pi)$, where $x_{\varepsilon}$ satisfies $\left|x_{\varepsilon}\right| \rightarrow a$, and $T_{\tau}$ denotes a continuous and fixed point free group action on A.

These solutions will be obtained by introducing a suitable group action $T_{\tau}$ on $A$; this symmetry is the natural one induced by the Hopf fibration of $S^{3}$. Then, looking for $T_{\tau^{-}}$ invariant solutions, one can reduce the problem to an equivalent nonhomogeneous equation in an annulus $B \subset \mathbb{R}^{3}$. To this equation the results of $\mathrm{Ni}$ and Wei [13] can be applied, producing single peak solutions. Adapting the methods of del Pino-Felmer [3] we show that the peaks converge to the inner boundary of $B$. Transforming back to the original problem then yields the result.

Our restriction to $\mathbb{R}^{4}$ is due to the fact that we can define an explicit fixed point free group action (see Remark B below). Since every smooth action on a $2 m$-sphere has fixed points, extensions of our result to odd dimensions seem impossible. On the other hand, extensions to higher even dimensions should be possible; however, since the explicit form of the reduced nonhomogeneous equation is required in order to study the behavior of the concentrating solutions, we need an explicit $2 m$-dimensional analogue of the coordinate system (4) (see below) together with a fixed point free action, as well as an explicit reduction procedure to an equation in $\mathbb{R}^{2 m-1}$; and this seems not easy to achieve.

Remark A. 1) Note that the natural limitation for $p$ due to the Sobolev embedding theorem in $\mathbb{R}^{4}$ is $1<p<(N+2) /(N-2)=3$; however, since by the above mentioned group invariance the problem will be reduced to a problem in three dimensions, we can allow $1<p<5$.

2) It is known that the single peak solutions for equation (1) concentrate at a point $P_{0}$ with $\left|P_{0}\right|=\max _{P \in A} d(P, \partial A)=(a+b) / 2$ (see [13]). On the other hand, the solutions concentrating on spheres found by Ambrosetti-Malchiodi-Ni [1] concentrate for $V(|x|) \equiv 1$ on the inner boundary of $A$ (since $\left.M(r)=r^{n-1}\right)$. Our result yields concentration orbits which also converge to the inner boundary of $A$. 


\section{The group action}

Consider the equation

$$
\begin{cases}-\varepsilon^{2} \Delta u+u=u^{p} & \text { in } A, \\ u=0 & \text { on } \partial A \\ u>0 & \text { in } A,\end{cases}
$$

where $A=\left\{x \in \mathbb{R}^{4}|0<a<| x \mid<b\right\}$ is an annular domain in $\mathbb{R}^{4}$, and $1<p<5$. Let $H_{0 \text {,rad }}^{1} \subset H_{0}^{1}(\Omega)$ denote the subspace of $H_{0}^{1}(\Omega)$ consisting of radial functions.

We consider the following coordinate system in $\mathbb{R}^{4}$ :

$$
\begin{array}{ll}
x_{1}=r \sin \theta_{1} \sin \theta_{3}, & a<r<b, \\
x_{2}=r \cos \theta_{1} \sin \theta_{3}, & 0 \leq \theta_{i}<2 \pi(i=1,2), \\
x_{3}=r \sin \theta_{2} \cos \theta_{3}, & 0 \leq \theta_{3}<\pi / 2, \\
x_{4}=r \cos \theta_{2} \cos \theta_{3}, &
\end{array}
$$

where $0 \leq \theta_{i} \leq 2 \pi(i=1,2)$ denote the angles between $\left(x_{1}, x_{2}\right)$ in the $x_{1} x_{2}$-plane and between $\left(x_{3}, x_{4}\right)$ in the $x_{3} x_{4}$-plane, and $0 \leq \theta_{3}<\pi / 2$ denotes the angle between the planes $x_{1} x_{2}$ and $x_{3} x_{4}$. A direct calculation gives the volume element in the $\left(r, \theta_{1}, \theta_{2}, \theta_{3}\right)$ coordinates:

$$
r^{3} \sin \theta_{3} \cos \theta_{3} d r d \theta_{1} d \theta_{2} d \theta_{3}
$$

A simple but tedious computation shows that the Laplacian $\Delta$ takes in the coordinate system (4) the form

$$
\begin{aligned}
\Delta u= & u_{r r}+\frac{3}{r} u_{r}+u_{\theta_{1} \theta_{1}} \frac{1}{r^{2} \sin ^{2} \theta_{3}}+u_{\theta_{2} \theta_{2}} \frac{1}{r^{2} \cos ^{2} \theta_{3}} \\
& +\frac{1}{r^{2}} u_{\theta_{3}}\left(-\frac{\sin \theta_{3}}{\cos \theta_{3}}+\frac{\cos \theta_{3}}{\sin \theta_{3}}\right)+\frac{1}{r^{2}} u_{\theta_{3} \theta_{3}} .
\end{aligned}
$$

Consider the following group action $T_{\tau}$ on $A$ : for

$$
z=\left(r, \theta_{1}, \theta_{2}, \theta_{3}\right), \quad a<r<b, \quad 0 \leq \theta_{i}<2 \pi(i=1,2), \quad 0 \leq \theta_{3}<\pi / 2,
$$

let

$$
T_{\tau} z=\left(r, \theta_{1}+\tau, \theta_{2}+\tau, \theta_{3}\right), \quad \tau \in[0,2 \pi),
$$

and define the subspace $H_{0, \#}^{1}(A) \subset H_{0}^{1}(A)$ of functions which are invariant under this action, i.e.

$$
u \in H_{0, \#}^{1} \quad \text { if } \quad u\left(T_{\tau} x\right)=u(x), \forall \tau \in[0,2 \pi) .
$$

Remark B. 1) Note that the action defined above is fixed point free. This is important, since otherwise the solutions might concentrate on fixed points and thus would not yield a concentrating orbit. 
2) The usual coordinate system in $\mathbb{R}^{4}$ is

$$
\begin{array}{ll}
x_{1}=r \sin \theta_{3} \sin \theta_{2} \sin \theta_{1}, & 0 \leq \theta_{1}<2 \pi, \\
x_{2}=r \sin \theta_{3} \sin \theta_{2} \cos \theta_{1}, & 0 \leq \theta_{2}<\pi, \\
x_{3}=r \sin \theta_{3} \cos \theta_{2}, & 0 \leq \theta_{3} \leq \pi, \\
x_{4}=r \cos \theta_{3}, & a<r<b .
\end{array}
$$

Since only the variable $\theta_{1}$ varies in $[0,2 \pi)$, the only obvious way to define a group action is $T_{\tau} u\left(r, \theta_{1}, \theta_{2}, \theta_{3}\right)=u\left(r, \theta_{1}+\tau, \theta_{2}, \theta_{3}\right)$. However, this action has the fixed points $x_{r}=$ $(0,0,0, r) \in \mathbb{R}^{4}, a<r<b$.

The following properties connected with the group action $T_{\tau}$ are easily verified:

Lemma 2. 1) $H_{0, \#}^{1}(A)$ is a closed subspace of $H_{0}^{1}(A)$.

2) $H_{0, \#}^{1}(A)$ is invariant under the Laplacian, i.e.

$$
u \in H_{0, \#}^{1}(A) \Rightarrow \Delta u \in H_{0, \#}^{1}(A) .
$$

3) $H_{0, \mathrm{rad}}^{1}(A) \subset H_{0, \#}^{1}(A)$.

For the moment, let us assume that $1<p<3$, so that the following functional $J(u)$ is well defined in $H_{0, \#}^{1}$ :

$$
J_{\varepsilon}(u)=\frac{\varepsilon^{2}}{2} \int_{\Omega}|\nabla u|^{2}+\frac{1}{2} \int_{\Omega} u^{2}-\frac{1}{p+1} \int_{\Omega}\left(u^{+}\right)^{p+1} .
$$

Using the Mountain-Pass Lemma [2], one finds a critical level for $J_{\varepsilon}(u)$, and one knows that at this level there exists a solution which has Morse index less than or equal to 1 (see [4, 7, 8]).

We now show that for $\varepsilon>0$ sufficiently small, this solution cannot be independent of the variable $\theta_{3}$.

Lemma 3. For $\varepsilon^{2}$ sufficiently small, the mountain pass solution $u_{\varepsilon}$ with Morse index 1 is not independent of the variable $\theta_{3}$.

Proof. Suppose to the contrary that $u_{\varepsilon}$ is independent of $\theta_{3}$. From

$$
-\varepsilon^{2} \Delta u_{\varepsilon}+u_{\varepsilon}-u_{\varepsilon}^{p}=0 \quad \text { in } A
$$

we get

$$
-\varepsilon^{2} \Delta u_{\varepsilon}-\left(p u_{\varepsilon}^{p-1}-1\right) u_{\varepsilon}=-(p-1) u_{\varepsilon}^{p} .
$$

On the left of the equation, we have the linearization of the operator in $(8)$ in direction $u_{\varepsilon}$, and hence we deduce from

$$
\left(-\varepsilon^{2} \Delta u_{\varepsilon}-\left(p u_{\varepsilon}^{p-1}-1\right) u_{\varepsilon}, u_{\varepsilon}\right)=-(p-1)\left(u_{\varepsilon}^{p}, u_{\varepsilon}\right)<0
$$

that $u_{\varepsilon}$ contributes 1 to the Morse index. 
Next, consider the function $u_{\varepsilon} \cos \left(2 \theta_{3}\right)$. Note that $u_{\varepsilon} \cos \left(2 \theta_{3}\right)$ is orthogonal to $u_{\varepsilon}$ on $A$, i.e.

$$
\int_{A} u_{\varepsilon} \cos \left(2 \theta_{3}\right) u_{\varepsilon} r^{3} \sin \theta_{3} \cos \theta_{3} d r d \theta_{1} d \theta_{2} d \theta_{3}=0
$$

since by our assumption $u_{\varepsilon}$ is independent of $\theta_{3}$ and since $\int_{0}^{\pi / 2} \cos \left(2 \theta_{3}\right) \sin \theta_{3} \cos \theta_{3} d \theta_{3}$ $=0$.

We now show that $u_{\varepsilon} \cos \left(2 \theta_{3}\right)$ also contributes 1 to the Morse index. We calculate $-\varepsilon^{2} \Delta\left[u_{\varepsilon} \cos \left(2 \theta_{3}\right)\right]$, using $[6$ :

$$
\begin{aligned}
-\varepsilon^{2} \Delta\left[u_{\varepsilon} \cos \left(2 \theta_{3}\right)\right]= & \left(u_{\varepsilon}^{p}-u_{\varepsilon}\right) \cos \left(2 \theta_{3}\right) \\
& -\frac{\varepsilon^{2}}{r^{2}} u_{\varepsilon}\left(-\frac{\sin \theta_{3}}{\cos \theta_{3}}+\frac{\cos \theta_{3}}{\sin \theta_{3}}\right)\left[\cos \left(2 \theta_{3}\right)\right]_{\theta_{3}}-\frac{\varepsilon^{2}}{r^{2}} u_{\varepsilon}\left[\cos \left(2 \theta_{3}\right)\right]_{\theta_{3} \theta_{3}} \\
= & \left(u_{\varepsilon}^{p}-u_{\varepsilon}\right) \cos \left(2 \theta_{3}\right)+\frac{4 \varepsilon^{2}}{r^{2}} u_{\varepsilon} \cos \left(2 \theta_{3}\right)+\frac{4 \varepsilon^{2}}{r^{2}} u_{\varepsilon} \cos \left(2 \theta_{3}\right) \\
= & \left(p u_{\varepsilon}^{p-1}-1\right) u_{\varepsilon} \cos \left(2 \theta_{3}\right)-(p-1) u_{\varepsilon}^{p} \cos \left(2 \theta_{3}\right)+\frac{8 \varepsilon^{2}}{r^{2}} u_{\varepsilon} \cos \left(2 \theta_{3}\right) .
\end{aligned}
$$

Multiplying by $u_{\varepsilon} \cos \left(2 \theta_{3}\right)$ and integrating over $A$, we see that the last two terms give

$$
\begin{aligned}
\int_{A} & \left(-(p-1) u_{\varepsilon}^{p} \cos \left(2 \theta_{3}\right)+\frac{8 \varepsilon^{2}}{r^{2}} u_{\varepsilon} \cos \left(2 \theta_{3}\right)\right) u_{\varepsilon} \cos \left(2 \theta_{3}\right) d x \\
= & \int_{0}^{\pi / 2} \frac{1}{2} \sin \left(2 \theta_{3}\right) \cos ^{2}\left(2 \theta_{3}\right) \int_{a}^{b} \int_{0}^{2 \pi} \int_{0}^{2 \pi}\left(-(p-1) u_{\varepsilon}^{p+1}+\frac{8 \varepsilon^{2}}{r^{2}} u_{\varepsilon}^{2}\right) d \theta_{1} d \theta_{2} r^{3} d r
\end{aligned}
$$

which is negative for $\varepsilon^{2}$ small, since $\int_{A} u_{\varepsilon}^{p+1}=\int_{A}\left(\varepsilon^{2}\left|\nabla u_{\varepsilon}\right|^{2}+u_{\varepsilon}^{2}\right)>\int_{A} u_{\varepsilon}^{2}$.

Thus, we have shown that any solution which is independent of $\theta_{3}$ has Morse index $\geq 2$; hence the mountain-pass solution, which has Morse index 1, cannot be independent of $\theta_{3}$.

We now use invariance under the group $T_{\tau}$ to reduce problem (3) to an equation in three dimensions.

\section{Reduction to a problem in three dimensions}

In the variables $\left(r, \theta_{1}, \theta_{2}, \theta_{3}\right)$ the first term of the functional $J_{\varepsilon}$ takes the form

$$
\begin{aligned}
\frac{\varepsilon^{2}}{2} \int_{0}^{\pi / 2} \int_{0}^{2 \pi} & \int_{0}^{2 \pi} \int_{a}^{b}\left(\left|u_{r}\right|^{2}+\left|u_{\theta_{1}}\right|^{2} \frac{1}{r^{2} \sin ^{2} \theta_{3}}\right. \\
& \left.+\left|u_{\theta_{2}}\right|^{2} \frac{1}{r^{2} \cos ^{2} \theta_{3}}+\left|u_{\theta_{3}}\right|^{2} \frac{1}{r^{2}}\right) r^{3} \sin \theta_{3} \cos \theta_{3} d r d \theta_{1} d \theta_{2} d \theta_{3} .
\end{aligned}
$$


We now rewrite the functional $J_{\varepsilon}(u)$, taking into account the invariance along the orbit $T_{\tau}$ : for $u \in H_{0, \#}^{1}(A)$ we can consider

$$
T_{-\theta_{2}} u\left(t, \theta_{1}, \theta_{2}, \theta_{3}\right)=u\left(r, \theta_{1}-\theta_{2}, 0, \theta_{3}\right)=: v\left(r, \theta, \theta_{3}\right),
$$

where we have introduced the new variable $\theta:=\theta_{1}-\theta_{2}$. Note that $v\left(r, \theta, \theta_{3}\right)$ is well defined: if $\theta=\theta_{1}^{\prime}-\theta_{2}^{\prime}$, then $\theta_{1}^{\prime}=\theta_{1}+\sigma$ for some $\sigma \in[0,2 \pi)$, and hence $\theta_{2}^{\prime}=\theta_{1}^{\prime}-\theta=$ $\theta_{1}+\sigma-\theta=\left(\theta_{2}+\theta\right)+\sigma-\theta=\theta_{2}+\sigma$.

Next, we calculate the derivative $v_{\theta}\left(r, \theta, \theta_{3}\right)$ : we have

$$
\begin{aligned}
& \lim _{h \rightarrow 0} \frac{1}{h}\left(v\left(r, \theta+h, \theta_{3}\right)-v\left(r, \theta, \theta_{3}\right)\right) \\
& \quad=\lim _{h \rightarrow 0} \frac{1}{h}\left(u\left(r, \theta_{1}+\frac{h}{2}, \theta_{2}-\frac{h}{2}, \theta_{3}\right)-u\left(r, \theta_{1}, \theta_{2}, \theta_{3}\right)\right) \\
& \quad=\lim _{h \rightarrow 0}\left(\frac{1}{h}\left(u\left(r, \theta_{1}+\frac{h}{2}, \theta_{2}-\frac{h}{2}, \theta_{3}\right)-u\left(r, \theta_{1}, \theta_{2}-\frac{h}{2}, \theta_{3}\right)\right)\right. \\
&\left.\quad+\frac{1}{h}\left(u\left(r, \theta_{1}, \theta_{2}-\frac{h}{2}, \theta_{3}\right)-u\left(r, \theta_{1}, \theta_{2}, \theta_{3}\right)\right)\right) \\
&=\frac{1}{2} u_{\theta_{1}}\left(r, \theta_{1}, \theta_{2}, \theta_{3}\right)-\frac{1}{2} u_{\theta_{2}}\left(r, \theta_{1}, \theta_{2}, \theta_{3}\right)=u_{\theta_{1}}\left(r, \theta_{1}, \theta_{2}, \theta_{3}\right)=-u_{\theta_{2}}\left(r, \theta_{1}, \theta_{2}, \theta_{3}\right) ;
\end{aligned}
$$

the last identities follow by considering the constant function

$$
f(\tau)=u\left(r, \theta_{1}+\tau, \theta_{2}+\tau, \theta_{3}\right),
$$

which yields

$$
0=f_{\tau}(0)=u_{\theta_{1}}\left(r, \theta_{1}, \theta_{2}, \theta_{3}\right)+u_{\theta_{2}}\left(r, \theta_{1}, \theta_{2}, \theta_{3}\right) .
$$

With this, we can now write the functional $J_{\varepsilon}$ on the space $H_{0, \#}^{1}(A)$ in the form

$$
\begin{aligned}
J_{\varepsilon}(v)=2 \pi \int_{0}^{\pi / 2} \int_{0}^{2 \pi} \int_{a}^{b}( & \frac{\varepsilon^{2}}{2}\left|v_{r}\right|^{2}+\frac{\varepsilon^{2}}{2}\left|v_{\theta}\right|^{2} \frac{1}{r^{2}}\left(\frac{1}{\sin ^{2} \theta_{3}}+\frac{1}{\cos ^{2} \theta_{3}}\right)+\frac{\varepsilon^{2}}{2}\left|v_{\theta_{3}}\right|^{2} \frac{1}{r^{2}} \\
& \left.+\frac{1}{2}|v|^{2}-\frac{1}{p+1}|v|^{p+1}\right) r^{3} \sin \theta_{3} \cos \theta_{3} d r d \theta d \theta_{3} .
\end{aligned}
$$

We make the change of variables $\varphi=2 \theta_{3}$, and use that $\sin \theta_{3} \cos \theta_{3}=\frac{1}{2} \sin \varphi$, to obtain

$$
\begin{array}{r}
J_{\varepsilon}(v)=2 \pi \int_{0}^{\pi} \int_{0}^{2 \pi} \int_{a}^{b}\left(\frac{\varepsilon^{2}}{2}\left|v_{r}\right|^{2}+\frac{\varepsilon^{2}}{2}\left|v_{\theta}\right|^{2} \frac{4}{r^{2} \sin ^{2} \varphi}+\frac{\varepsilon^{2}}{2}\left|v_{\varphi}\right|^{2} \frac{4}{r^{2}}\right. \\
\left.+\frac{1}{2}|v|^{2}-\frac{1}{p+1}|v|^{p+1}\right) \frac{r^{3}}{4} \sin \varphi d r d \theta d \varphi \\
=2 \pi \int_{0}^{\pi} \int_{0}^{2 \pi} \int_{a}^{b}\left(\frac{\varepsilon^{2}}{2}\left|v_{r}\right|^{2} \frac{r^{3} d r}{4}+\frac{\varepsilon^{2}}{2}\left|v_{\theta}\right|^{2} \frac{r d r}{\sin ^{2} \varphi}+\frac{\varepsilon^{2}}{2}\left|v_{\varphi}\right|^{2} r d r\right. \\
\left.+\frac{1}{2}|v|^{2} \frac{r^{3} d r}{4}-\frac{1}{p+1}|v|^{p+1} \frac{r^{3} d r}{4}\right) \sin \varphi d \theta d \varphi .
\end{array}
$$


Now, setting $s=\frac{1}{2} r^{2}$ we get

$$
\begin{aligned}
J_{\varepsilon}(v)=2 \pi \int_{0}^{\pi} \int_{0}^{2 \pi} \int_{a^{2} / 2}^{b^{2} / 2}\left(\frac{\varepsilon^{2}}{2}\left|v_{s}\right|^{2} s^{2} d s\right. & +\frac{\varepsilon^{2}}{2}\left|v_{\theta}\right|^{2} \frac{d s}{\sin ^{2} \varphi}+\frac{\varepsilon^{2}}{2}\left|v_{\varphi}\right|^{2} d s \\
& \left.+\frac{1}{2}|v|^{2} \frac{s d s}{2}-\frac{1}{p+1}|v|^{p+1} \frac{s d s}{2}\right) \sin \varphi d \theta d \varphi \\
=2 \pi \int_{0}^{\pi} \int_{0}^{2 \pi} \int_{a^{2} / 2}^{b^{2} / 2}\left(\frac{\varepsilon^{2}}{2}\left|v_{s}\right|^{2}+\right. & \frac{\varepsilon^{2}}{2}\left|v_{\theta}\right|^{2} \frac{1}{s^{2} \sin ^{2} \varphi}+\frac{\varepsilon^{2}}{2}\left|v_{\varphi}\right|^{2} \frac{1}{s^{2}} \\
& \left.+\frac{1}{2}|v|^{2} \frac{1}{2 s}-\frac{1}{p+1}|v|^{p+1} \frac{1}{2 s}\right) s^{2} d s \sin \varphi d \theta d \varphi .
\end{aligned}
$$

We note that this functional is now defined in the usual polar coordinates $(r, \theta, \varphi)$ in $\mathbb{R}^{3}$, and we may rewrite it after a standard change of variables in cartesian coordinates:

$$
J_{\varepsilon}(v)=\int_{B}\left(\frac{\varepsilon^{2}}{2}|\nabla v|^{2}+\frac{1}{2}|v|^{2} \frac{1}{2|x|}-\frac{1}{p+1}|v|^{p+1} \frac{1}{2|x|}\right) d x
$$

where $B=\left\{x \in \mathbb{R}^{3}: a^{2} / 2<|x|<b^{2} / 2\right\}$. Critical points of this functional now correspond to the following "reduced" equation in $B \subset \mathbb{R}^{3}$ :

$$
\begin{cases}-\varepsilon^{2} \Delta v+\frac{1}{2|x|} v-\frac{1}{2|x|} v^{p}=0 & \text { in } B, \\ v=0 & \text { on } \partial B .\end{cases}
$$

It is clear that if we have a solution of equation 12 in $B \subset \mathbb{R}^{3}$, then by reversing the above transformations, we will obtain a solution of equation $\sqrt{3}$ in $A \subset \mathbb{R}^{4}$. We remark in particular that if we find, for $\varepsilon^{2}$ small, solutions $v_{\varepsilon}$ which converge to a single peak solution of equation (12), then the corresponding solutions $u_{\varepsilon}$ will concentrate on a 1-dimensional curve given by the orbit under $T_{\tau}$ of $v_{\varepsilon}$.

\section{Profile of the solution}

W.-M. Ni and J. Wei studied in [13] the equation

$$
\left\{\begin{array}{l}
-\varepsilon^{2} \Delta u+u=u^{p} \quad \text { in } \Omega \subset \mathbb{R}^{n}, \\
u>0 \quad \text { in } \Omega, \quad u=0 \quad \text { on } \partial \Omega,
\end{array}\right.
$$

and proved (Theorem 2.2 in [13]) that the least energy solutions of $[13$ have, for $\varepsilon>0$ sufficiently small, at most one local maximum. This result is proved by a blow-up procedure which leads to the following limiting equation in $\mathbb{R}^{n}$ :

$$
\left\{\begin{array}{l}
-\Delta z+z-z^{p}=0 \text { on } \mathbb{R}^{n} \\
z(x) \rightarrow 0 \text { as }|x| \rightarrow \infty
\end{array}\right.
$$

The following proposition is an adaptation of the above mentioned theorem by $\mathrm{Ni}$ and Wei to the nonhomogeneous equation (12). 
Proposition 4. Let $u_{\varepsilon}$ be a least energy solution to 12 . Then, for $\varepsilon^{2}$ sufficiently small:

(i) $u_{\varepsilon}$ has at most one local maximum and it is achieved at exactly one point $p_{\varepsilon}$ in $B$. Moreover, $u_{\varepsilon}\left(\cdot+p_{\varepsilon}\right) \rightarrow 0$ in $C_{\mathrm{loc}}^{1}\left(\left(B-p_{\varepsilon}\right) \backslash\{0\}\right)$ where $B-p_{\varepsilon}=\left\{x-p_{\varepsilon} \mid x \in B\right\}$.

(ii) $(1 / \varepsilon) d\left(p_{\varepsilon}, \partial B\right) \rightarrow+\infty$ as $\varepsilon \rightarrow \infty$.

The blow-up procedure leads in this case to the limit equation

$$
\left\{\begin{array}{l}
-\Delta w+\frac{1}{2 d} w-\frac{1}{2 d} w^{p}=0 \quad \text { in } \mathbb{R}^{3}, \\
w(x) \rightarrow 0 \text { as }|x| \rightarrow \infty
\end{array}\right.
$$

with $d=\left|p_{0}\right|$.

Following the work of del Pino-Felmer [3], who simplified the proof of Ni-Wei (and at the same time removed a delicate "nondegeneracy condition"), we will prove

Theorem 5. For $\varepsilon \rightarrow 0$ the maximum point $p_{\varepsilon}$ converges to the inner boundary of $B$, i.e.

$$
\left|p_{\varepsilon}\right| \rightarrow a^{2} / 2 \quad \text { as } \varepsilon \rightarrow 0 .
$$

This is in contrast to the homogeneous equation (13) for which Ni-Wei proved concentration at a point with maximal distance from the boundary.

\section{Concentrating solutions}

In this section we point out the necessary modifications in the proof of Theorem 2.2 by $\mathrm{Ni}$ and Wei when applied to the nonhomogeneous equation (12) to obtain concentrating solutions.

1) Proof of Proposition 4. This corresponds to the proof of Theorem 2.2(i) in Ni-Wei [13]. Lemmas 3.1-3.3 there require no change.

Step 1 (p. 737). We first prove (ii). Assume on the contrary that there exists $c>0$ and a sequence $\varepsilon_{k} \rightarrow 0$ such that

$$
d\left(p_{\varepsilon}, \partial B\right) \leq c \varepsilon \quad \text { for } \varepsilon=\varepsilon_{k} .
$$

By passing to a subsequence, we may assume that $p_{\varepsilon} \rightarrow p_{0} \in \partial B$, i.e. $\left|p_{0}\right|=a^{2} / 2$ or $\left|p_{0}\right|=b^{2} / 2$. Writing $p_{\varepsilon}=p_{0}+\widetilde{p}_{\varepsilon}$, we have $\widetilde{p}_{\varepsilon} \rightarrow 0$. By "boundary straightening" around the point $p_{0}$ we obtain for $w_{\varepsilon}(z):=u_{\varepsilon}\left(\mathcal{G}\left(q_{\varepsilon}+\varepsilon z\right)\right)$ the equation

$$
\sum_{i, j} a_{i, j}^{\varepsilon} \frac{\partial^{2} w_{\varepsilon}}{\partial z_{i} \partial z_{j}}+\varepsilon \sum_{i} b_{j}^{\varepsilon} \frac{\partial w_{\varepsilon}}{\partial z_{j}}-\frac{w_{\varepsilon}}{2\left|p_{0}+\mathcal{G}\left(q_{\varepsilon}+\varepsilon z\right)\right|}+\frac{w_{\varepsilon}^{p}}{2\left|p_{0}+\mathcal{G}\left(q_{\varepsilon}+\varepsilon z\right)\right|},
$$

where $\mathcal{G}: \bar{B}_{\kappa / \varepsilon} \cap\left\{z_{3} \geq-\alpha_{\varepsilon}\right\} \subset \mathbb{R}^{3} \rightarrow B$ is the "straightening map" with $\mathcal{G}\left(q_{\varepsilon}\right)=p_{\varepsilon}$; $\kappa>0$ is a small constant and $\alpha_{\varepsilon}>0$ is bounded. In the limit $\varepsilon \rightarrow 0$ we find, since 
$q_{\varepsilon} \rightarrow 0$, that $w_{0}=\lim w_{\varepsilon}$ satisfies the equation (cf. (3.7) in [13])

$$
\left\{\begin{array}{l}
\Delta u-\frac{u}{2\left|p_{0}\right|}+\frac{u^{p}}{2\left|p_{0}\right|}=0, \quad u>0, \quad \text { on } \mathbb{R}_{\alpha,+}^{3}, \\
u=0 \quad \text { on } \partial \mathbb{R}_{\alpha,+}^{3},
\end{array}\right.
$$

where $\mathbb{R}_{\alpha,+}^{3}=\left\{z \in \mathbb{R}^{3} \mid z_{3} \geq-\alpha\right\}$ with $\alpha=\lim \alpha_{\varepsilon}$.

By Theorem 1.1 of [6] one concludes that $w_{0} \equiv 0$; but this contradicts that $w_{0}(0)=$ $\lim w_{\varepsilon}\left(q_{\varepsilon}\right)=\lim u_{\varepsilon}\left(p_{\varepsilon}\right) \geq \bar{u}$.

Steps 2 and 3 are the same, provided Proposition 3.4 works (see below). This gives the conclusion of Theorem 2.2(i) of [13].

2) Profile of $u_{\varepsilon}$. The statement and proof of Proposition 3.4 in [13] need some modifications. For convenience, we state it here:

Proposition 3.4'. Let $\tilde{v}_{\varepsilon}(y)=u_{\varepsilon}\left(p_{\varepsilon}+\varepsilon y\right)$.

(i) For given $\eta>0$ there exist $\varepsilon_{0}>0$ and $k_{0}>0$ such that for $0<\varepsilon<\varepsilon_{0}$,

$$
B_{2 k_{0} \varepsilon}\left(p_{\varepsilon}\right) \subset B \text { and }\left\|\tilde{v}_{\varepsilon}-w_{d}\right\|_{C^{2}\left(B_{k_{0}}(0)\right)}<\eta,
$$

where $w_{d}$ is the unique solution of equation [15, with

$$
w_{d}(x):=w\left(\frac{x}{\sqrt{2 d}}\right)
$$

and $w$ the unique solution of 144 satisfying $w(0)=\max _{x \in \mathbb{R}^{3}} w(x)$ and $w(x) \rightarrow 0$ as $|x| \rightarrow \infty$.

(ii) For any $0<\delta<1$ there exists a constant $C$ such that

$$
\tilde{v}_{\varepsilon}(y) \leq C e^{-\frac{\sqrt{1-\delta}}{b}|y|} \quad \text { for } y \in \widetilde{B}_{\varepsilon}:=\left\{y \in \mathbb{R}^{3} \mid p_{\varepsilon}+\varepsilon y \in B\right\},
$$

where $b$ is the outer radius of the annulus $A$.

Proof. (i): As in Ni-Wei [13].

(ii): Comparison with the known solution $w_{d}(r)$ of equation (15): By Theorem 2 in Gidas-Ni-Nirenberg [9] one knows that for the solution $w(r)$ of (14] one has $w(r) \leq$ $C_{0} e^{-r}$, and hence by 16 ,

$$
w_{d}(r) \leq C_{0} e^{-r / \sqrt{2 d}} \quad \text { for } r \geq 0 .
$$

For given $\eta>0$ choose $R>0$ such that $\eta=C_{0} e^{-R / \sqrt{2 d}}$. By (i) there exists $\varepsilon_{0}>0$ such that $\left\|\tilde{v}_{\varepsilon}-w_{d}\right\|_{C^{2}\left(\bar{B}_{2 R}(0)\right)} \leq \eta$ for $0<\varepsilon<\varepsilon_{0}$. Thus

$$
\tilde{v}_{\varepsilon}(y) \leq w(y)+\eta \leq C_{0} e^{-R / \sqrt{2 d}}+\eta=2 \eta \quad \text { for }|y|=R .
$$


Hence (see Ni-Wei [13])

$$
v_{\varepsilon} \leq 2 \eta \quad \text { in } B_{\varepsilon}^{(o)}:=B \backslash B_{R \varepsilon}\left(p_{\varepsilon}\right) .
$$

Note that $\tilde{v}_{\varepsilon}$ satisfies

$$
\left\{\begin{array}{l}
\Delta \tilde{v}_{\varepsilon}-\frac{1}{2\left|p_{\varepsilon}+\varepsilon y\right|}\left(1-\tilde{v}_{\varepsilon}^{p-1}\right) \tilde{v}_{\varepsilon}=0 \quad \text { in } \widetilde{B}_{\varepsilon}^{(o)}:=\widetilde{B}_{\varepsilon} \backslash B_{R}(0), \\
\left.\tilde{v}_{\varepsilon}\right|_{\partial B_{R}(0) \leq 2 \eta,} \tilde{v}_{\varepsilon}=0 \quad \text { on } \partial \widetilde{B}_{\varepsilon}
\end{array}\right.
$$

and that $\left|p_{\varepsilon}+\varepsilon y\right| \leq b^{2} / 2$. Now choose $\eta$ such that $1-s^{p-1}>1-\delta$ for $s<2 \eta$, and hence

$$
\frac{1}{2\left|p_{\varepsilon}+\varepsilon y\right|}\left(1-\tilde{v}_{\varepsilon}^{p-1}\right)>\frac{1}{b^{2}}(1-\delta) \quad \text { in } \widetilde{B}_{\varepsilon}^{(o)} .
$$

Let $G_{0}(|y|)$ denote the Green's function for $-\Delta+1$ on $\mathbb{R}^{3}$, and

$$
\bar{v}(y)=\frac{2 \eta G_{0}(|y| \sqrt{1-\delta} / b)}{G_{0}(R \sqrt{1-\delta} / b)} .
$$

Then $\bar{v}$ satisfies

$$
\Delta \bar{v}-\frac{1-\delta}{b^{2}} \bar{v}=0, \quad \bar{v}=2 \eta \quad \text { on } \partial B_{R}(0)
$$

By the maximum principle on $\widetilde{B}_{\varepsilon}^{(o)}$ we have

$$
\tilde{v}_{\varepsilon}(y) \leq \bar{v}(y) \quad \text { on } \widetilde{B}_{\varepsilon}^{(o)},
$$

and hence

$$
\tilde{v}_{\varepsilon}(y) \leq C e^{-\frac{\sqrt{1-\delta}}{b}|y|} \quad \text { for all } y \in \widetilde{B}_{\varepsilon}^{(o)} .
$$

\section{Localizing the concentration points}

In this section we show that the concentration points $p_{\varepsilon}$ for the least energy solution of the nonhomogeneous equation 12 converge to the inner boundary of $B$, i.e. they satisfy

$$
\left|p_{\varepsilon}\right| \rightarrow a^{2} / 2 \quad \text { as } \varepsilon \rightarrow 0 \text {. }
$$

We follow the paper of del Pino-Felmer [3], obtaining precise upper and lower estimates for the least energy level $c_{\varepsilon}$.

\subsection{Upper bound}

Consider

$$
I_{d}\left(w_{d}\right)=\frac{1}{2} \int_{\mathbb{R}^{3}}\left|\nabla w_{d}\right|^{2} d x+\frac{1}{2 d} \int_{\mathbb{R}^{3}} \frac{1}{2}\left|w_{d}\right|^{2} d x-\frac{1}{2 d} \int_{\mathbb{R}^{3}} \frac{1}{p+1}\left|w_{d}\right|^{p+1} d x
$$


where $w_{d}$ is the solution of $[15]$, i.e.

$$
\left\{\begin{array}{l}
-\Delta w+\frac{1}{2 d} w-\frac{1}{2 d} w^{p}=0 \text { in } \mathbb{R}^{3}, \\
w(x) \rightarrow 0 \text { as }|x| \rightarrow \infty
\end{array}\right.
$$

We first note that

$$
I_{d}\left(w_{d}\right)=\sqrt{2 d} I(z)
$$

where $z$ is the solution of the equation

$$
-\Delta z+z-z^{p}=0 \quad \text { on } \mathbb{R}^{3},
$$

and $I(z)$ the corresponding functional. Note that the unique radial solution of (17) is given by

$$
w(r)=z\left(\frac{r}{\sqrt{2 d}}\right) .
$$

Then we calculate, denoting by $\omega_{2}$ the surface volume of $S^{2} \subset \mathbb{R}^{3}$ :

$$
\begin{aligned}
I_{d}\left(w_{d}\right)= & \frac{1}{2} \int\left|\nabla w_{d}\right|^{2}-\frac{1}{2 d} \int \frac{1}{2}\left|w_{d}\right|^{2}-\frac{1}{2 d} \int \frac{1}{p+1}\left|w_{d}\right|^{p+1} \\
= & \frac{\omega_{2}}{2} \int_{0}^{\infty}\left|\nabla_{r} z\left(\frac{r}{\sqrt{2 d}}\right)\right|^{2} r^{2} d r-\frac{\omega_{2}}{2} \frac{1}{2 d} \int_{0}^{\infty}\left|z\left(\frac{r}{\sqrt{2 d}}\right)\right|^{2} r^{2} d r \\
& -\frac{\omega_{2}}{p+1} \frac{1}{2 d} \int_{0}^{\infty}\left|z\left(\frac{r}{\sqrt{2 d}}\right)\right|^{p+1} r^{2} d r \\
= & \frac{\omega_{2}}{2} \int_{0}^{\infty}\left|\nabla_{s} z(s) \frac{d s}{d r}\right|^{2} 2 d \sqrt{2 d} s^{2} d s-\frac{\omega_{2}}{2} \frac{1}{\sqrt{2 d}} \int_{0}^{\infty}|z(s)|^{2} 2 d \sqrt{2 d} s^{2} d s \\
& -\frac{\omega_{2}}{p+1} \frac{1}{2 d} \int_{0}^{\infty}|z(s)|^{p+1} 2 d \sqrt{2 d} s^{2} d s \\
= & \sqrt{2 d} I(z) .
\end{aligned}
$$

Next, we derive an upper bound on the least energy value $c_{\varepsilon}$.

Lemma 6. Denote by $u_{\varepsilon}$ the minimal energy solution of equation $[12$, with the corresponding functional

$$
I_{\varepsilon,|x|}(u)=\frac{\varepsilon^{2}}{2} \int_{B}|\nabla u|^{2}+\frac{1}{2} \int_{B} \frac{1}{2|x|}|u|^{2}-\frac{1}{p+1} \int_{B} \frac{1}{2|x|}|u|^{p+1}
$$

and set

$$
c_{\varepsilon}=I_{\varepsilon,|x|}\left(u_{\varepsilon}\right)
$$

Then, for given $\delta>0$,

$$
c_{\varepsilon} \leq \varepsilon^{3}\left(\sqrt{a^{2}+\delta} I(z)+\varepsilon c\right) \quad \text { for } \varepsilon>0 \text { sufficiently small. }
$$


Proof. Consider a point $x_{\varepsilon}$ which lies close to the inner boundary, i.e. with $\operatorname{dist}\left(x_{\varepsilon}, a^{2} / 2\right)$ $=\delta / 2$, and the ball $B_{\delta / 2}\left(x_{\varepsilon}\right)$. Since the least energy values for $I_{\varepsilon,|x|}$ in $B$ and $B_{\delta / 2}\left(x_{\varepsilon}\right) \subset$ $B$ are ordered with respect to the domain we get

$$
c_{\varepsilon} \leq I_{\varepsilon,|x|}\left(u_{\delta / 2}\right)=: c_{\varepsilon, \delta / 2},
$$

where $u_{\delta / 2}$ is the least energy solution on $B_{\delta / 2}\left(x_{\varepsilon}\right)$. Recall that

$$
I_{\varepsilon,|x|}\left(u_{\delta / 2}\right)=\inf _{u \in S_{\varepsilon,|x|}} I_{\varepsilon,|x|}(u),
$$

where

$$
S_{\varepsilon,|x|}=\left\{u \in H_{0}^{1}\left(B_{\delta / 2}\left(x_{\varepsilon}\right)\right) \mid \int_{B_{\delta / 2}\left(x_{\varepsilon}\right)}\left(\varepsilon^{2}|\nabla u|^{2}+\frac{|u|^{2}}{2|x|}\right)=\int_{B_{\delta / 2}\left(x_{\varepsilon}\right)} \frac{|u|^{p+1}}{2|x|}\right\} .
$$

Next, let $\tilde{u}_{\delta / 2}$ denote the least energy solution of

$$
-\varepsilon^{2} \Delta u+\frac{1}{2 d_{\varepsilon}} u=\frac{1}{2 d_{\varepsilon}} u^{p} \quad \text { on } B_{\delta / 2}\left(x_{\varepsilon}\right),
$$

where $d_{\varepsilon}=\left|x_{\varepsilon}\right|$. Then $\tilde{u}_{\delta / 2}$ is radially symmetric, and by 20 ,

$$
I_{\varepsilon,|x|}\left(u_{\delta / 2}\right) \leq I_{\varepsilon,|x|}\left(\tilde{t}_{\delta / 2}\right),
$$

where $\tilde{t}$ is such that $\tilde{t} \tilde{u}_{\delta / 2} \in S_{\varepsilon,|x|}$. We estimate

$$
\begin{aligned}
I_{\varepsilon,|x|}\left(\tilde{t}_{\delta / 2}\right) & \tilde{\varepsilon}^{2} \\
= & \int_{B_{\delta / 2}\left(x_{\varepsilon}\right)}\left|\nabla \tilde{t} \tilde{u}_{\delta / 2}\right|^{2}+\frac{1}{2} \int_{B_{\delta / 2}\left(x_{\varepsilon}\right)} \frac{\left|\tilde{t} \tilde{u}_{\delta / 2}\right|^{2}}{2\left|x_{\varepsilon}+\left(x-x_{\varepsilon}\right)\right|} \\
& -\frac{1}{p+1} \int_{B_{\delta / 2}\left(x_{\varepsilon}\right)} \frac{\left|\tilde{t} \tilde{u}_{\delta / 2}\right|^{p+1}}{2\left(\left|x_{\varepsilon}+\left(x-x_{\varepsilon}\right)\right|\right)} \\
\leq & \frac{\varepsilon^{2}}{2} \int_{B_{\delta / 2}\left(x_{\varepsilon}\right)}\left|\nabla \tilde{t} \tilde{u}_{\delta / 2}\right|^{2}+\frac{1}{2} \int_{B_{\delta / 2}\left(x_{\varepsilon}\right)} \frac{\left|\tilde{t} \tilde{u}_{\delta / 2}\right|^{2}}{2\left|x_{\varepsilon}\right|-2\left|x-x_{\varepsilon}\right|} \\
& -\frac{1}{p+1} \int_{B_{\delta / 2}\left(x_{\varepsilon}\right)} \frac{\left|\tilde{t} \tilde{u}_{\delta / 2}\right|^{p+1}}{2\left|x_{\varepsilon}\right|+2\left|x-x_{\varepsilon}\right|}\left|\tilde{t} \tilde{u}_{\delta / 2}\right|^{p+1} \\
= & \frac{\varepsilon^{2}}{2} \int_{B_{\delta / 2}\left(x_{\varepsilon}\right)}\left|\nabla \tilde{t} \tilde{u}_{\delta / 2}\right|^{2}+\frac{1}{2} \int_{B_{\delta / 2}\left(x_{\varepsilon}\right)} \frac{\left|\tilde{t} \tilde{u}_{\delta / 2}\right|^{2}}{2\left|x_{\varepsilon}\right|}-\frac{1}{p+1} \int_{B_{\delta / 2}\left(x_{\varepsilon}\right)} \frac{1}{\left|\tilde{t} \tilde{u}_{\delta / 2}\right|^{2}\left|x-x_{\varepsilon}\right|} \\
& +\frac{1}{2} \int_{B_{\delta / 2}\left(x_{\varepsilon}\right)} \frac{\left.1 \tilde{u}_{\delta / 2}\right|^{p+1}\left|x-x_{\varepsilon}\right|}{2\left|x_{\varepsilon}\right|\left|x-x_{\varepsilon}\right|} .
\end{aligned}
$$

We now consider the problem

$$
-\Delta u+\frac{1}{2 d_{\varepsilon}} u=\frac{1}{2 d_{\varepsilon}} u^{p} \quad \text { in } B_{\rho}, \quad u>0 \quad \text { in } B_{\rho}, \quad u=0 \quad \text { on } \partial B_{\rho},
$$

with the associated functional $J_{\rho, d_{\varepsilon}}: H_{0}^{1}\left(B_{\rho}\right) \rightarrow \mathbb{R}$, where $d_{\varepsilon}=\left|x_{\varepsilon}\right|$. 
With the change of variable $x=x_{\varepsilon}+\varepsilon y$, setting $\rho=\delta / 2 \varepsilon$ and $v_{\delta / 2}(y)=\tilde{t} \tilde{u}_{\delta / 2}\left(x_{\varepsilon}+\right.$ $\varepsilon y)$, we obtain from (21)

$$
\begin{aligned}
I_{\varepsilon,|x|}\left(\tilde{t} \tilde{u}_{\delta / 2}\right) & \leq \varepsilon^{3}\left(J_{\rho, d_{\varepsilon}}\left(v_{\delta / 2}\right)+\varepsilon c \int_{B_{\rho}\left(x_{\varepsilon}\right)}\left|v_{\delta / 2}\right|^{2}|y| d y+\varepsilon c \int_{B_{\rho}\left(x_{\varepsilon}\right)}\left|v_{\delta / 2}\right|^{p+1}|y| d y\right) \\
& \leq \varepsilon^{3}\left(J_{\rho, d_{\varepsilon}}\left(v_{\delta / 2}\right)+\varepsilon c\right) \leq \varepsilon^{3}\left(J_{\rho, d_{\varepsilon}}\left(\bar{t} v_{\delta / 2}\right)+\varepsilon c\right),
\end{aligned}
$$

where $\bar{t}$ is such that $\bar{t} v_{\delta / 2} \in S_{\rho,\left|x_{\varepsilon}\right|}$, i.e. $\bar{t} v_{\delta / 2}$ solves 22]. Next, we use Lemma 2.1 in [3] to get

$$
J_{\rho, d_{\varepsilon}}\left(\bar{t} v_{\delta / 2}\right) \leq I_{d_{\varepsilon}}(w)+e^{-2 \rho(1+o(1))}=\sqrt{2 d_{\varepsilon}} I(z)+e^{-\frac{\delta}{\varepsilon}(1+o(1))},
$$

and hence finally

$$
c_{\varepsilon} \leq \varepsilon^{3}\left(\sqrt{2 d_{\varepsilon}} I(z)+\varepsilon c\right)=\varepsilon^{3}\left(\sqrt{a^{2}+\delta} I(z)+\varepsilon c\right) .
$$

\subsection{Lower bound}

In this section we show

Lemma 7. Let $p_{\varepsilon}$ denote the maximum point of the least energy solution $u_{\varepsilon}$ of equation 12). If $\left|p_{\varepsilon}\right| \geq a^{2} / 2+\delta$ for all $\varepsilon>0$, then there exists a positive constant (independent of $\varepsilon$ ) such that

$$
c_{\varepsilon} \geq \varepsilon^{3}\left(\sqrt{a^{2}+2 \delta} I(z)-\varepsilon c\right) .
$$

Proof. First note that

$$
c_{\varepsilon}=\max _{t>0} I_{\varepsilon,|x|}\left(t u_{\varepsilon}\right) \geq I_{\varepsilon,|x|}\left(t u_{\varepsilon}\right) \quad \text { for (say) } t \in[0,2] .
$$

Consider $B_{\delta}\left(p_{\varepsilon}\right) \subset B$, and fix $0<\delta^{\prime}<\delta$. Setting $x=p_{\varepsilon}+\varepsilon y, \rho=\delta / \varepsilon, v_{\varepsilon}(y)=$ $u_{\varepsilon}\left(p_{\varepsilon}+\varepsilon y\right)$ and $J_{\rho, d_{\varepsilon}}$ as in (22), we obtain, by a similar estimate to (21),

$$
I_{\varepsilon,|x|}\left(t u_{\varepsilon}\right) \geq \varepsilon^{3}\left(J_{\rho, d_{\varepsilon}}\left(t v_{\varepsilon}\right)-\varepsilon c\right) .
$$

Next, choose $\eta \in C^{1}\left([0, \delta], \mathbb{R}^{+}\right)$with $\eta(s)=1$ for $0 \leq s \leq \delta^{\prime}$ and $\eta(s)=0$ for $s \geq \delta$, and such that $\left|\eta^{\prime}(s)\right| \leq c$. Set $\tilde{v}_{\varepsilon}(y)=v_{\varepsilon}(y) \eta(|y|)$. Then, as for (2.6) in [3], we get

$$
\varepsilon^{3}\left(J_{\rho, d_{\varepsilon}}\left(t v_{\varepsilon}\right)-\varepsilon c\right) \geq \varepsilon^{3}\left(J_{\rho, d_{\varepsilon}}\left(t \tilde{v}_{\varepsilon}\right)-e^{-2 \delta^{\prime} / \varepsilon}-\varepsilon c\right) .
$$

At this point we choose $t=t^{*}$ such that $J_{\rho, d_{\varepsilon}}\left(t^{*} v_{\varepsilon}\right)=\max _{t \geq 0} J_{\rho, d_{\varepsilon}}\left(t v_{\varepsilon}\right)$, and conclude that

$$
J_{\rho, d_{\varepsilon}}\left(t^{*} v_{\varepsilon}\right) \geq J_{\rho, d_{\varepsilon}}\left(w_{\varepsilon}\right), \quad \text { where } w_{\varepsilon} \text { solves (22). }
$$

Finally, using the estimate of Lemma 2.1 in [3], we get

$$
J_{\rho, d_{\varepsilon}}\left(w_{\varepsilon}\right) \geq I_{d_{\varepsilon}}(w)-e^{-2 \delta^{\prime} / \varepsilon} .
$$

Joining the above estimates we obtain

$$
c_{\varepsilon} \geq \varepsilon^{3}\left(I_{d_{\varepsilon}}(w)-c \varepsilon\right)=\varepsilon^{3}\left(\sqrt{a^{2}+2 \delta} I(z)-c \varepsilon\right) .
$$




\section{Proof of Theorem 1}

From Lemmas 6 and 7 it follows that, for $\varepsilon>0$ sufficiently small, one has

$$
\left|p_{\varepsilon}\right| \leq a^{2} / 2+\delta
$$

Since $\delta>0$ is arbitrary, we conclude that $\left|p_{\varepsilon}\right|$ converges to the inner boundary of $B$. Note that Theorem 4 says that this convergence cannot be too fast, since

$$
\frac{1}{\varepsilon} d\left(p_{\varepsilon}, \partial B\right) \rightarrow+\infty \text {. }
$$

Finally, reversing the steps in Section 3 we see that the solutions $u_{\varepsilon}$ concentrating on the single peak $p_{\varepsilon}$, which we obtained in the previous sections, become solutions which concentrate on the orbits $T_{\tau} p_{\varepsilon}$ of the group action $T_{\tau}$ which converge to the inner boundary $a$ of $A$ for $\varepsilon \rightarrow 0$.

Acknowledgments. The authors wish to thank the referee for his very helpful comments.

\section{References}

[1] Ambrosetti, A., Malchiodi, A., Ni, W.-N.: Singularly perturbed elliptic equations with symmetry: existence of solutions concentrating on spheres, part II. Indiana Univ. Math. J. 53, 297-329 (2004) Zbl 1081.35008 MR 2056434

[2] Ambrosetti, A., Rabinowitz, P. H.: Dual variational methods in critical point theory and applications. J. Funct. Anal. 14, 349-381 (1973) Zbl 0273.49063 MR 0370183

[3] del Pino, M., Felmer, P.: Spike-layered solutions of singularly perturbed elliptic problems in a degenerate setting. Indiana Univ. Math. J. (2004) 48, 883-898 (1999) Zbl 0932.35080 MR 1736974

[4] Ekeland, I., Ghoussoub, N.: Selected new aspects of the calculus of variations in the large. Bulll. Amer. Math. Soc. (N.S.) 39, 207-265 (2002) Zbl 1064.35054 MR 1886088

[5] Esposito, P., Mancini, G., Santra, S., Srikanth, P. N.: Asymptotic behavior of radial solutions for a semilinear elliptic problem on an annulus through Morse index. J. Differential Equations 239, 1-15 (2007) Zbl 1143.35039 MR 2341546

[6] Esteban, M. J., Lions, P.-L.: Existence and nonexistence results for semilinear elliptic problems in unbounded domains. Proc. Roy Soc. Edinburgh Sect. A 93, 1-14 (1982) Zbl 0506.35035 MR 0688279

[7] Fang, G., Ghoussoub, N.: Morse-type information on Palais-Smale sequences obtained by Min-Max principles. Comm. Pure Appl. Math. 47, 1595-1653 (1994) Zbl 0829.58008 MR 1303222

[8] Ghoussoub, N.: Duality and Perturbation Methods in Critical Point Theory. Cambridge Tracts in Math. 107, Cambridge Univ. Press (1993) Zbl 0790.58002 MR 1251958

[9] Gidas, B., Ni, W.-M., Nirenberg, L.: Symmetry of positive solutions of nonlinear elliptic equations in $\mathbb{R}^{n}$. In: Mathematical Analysis and Applications, Adv. Math. Suppl. Stud. 7A, Academic Press, 369-402 (1981) Zbl 0469.35052 MR 0634248

[10] Lin, C. S., Ni, W.-M., Takagi, I.: Large amplitute stationary solutions to a chemotaxis system. J. Differential Equations 72, 1-27 (1988) Zbl 0676.35030 MR 0929196 
[11] Ni, W.-M., Takagi, I.: On the shape of least energy solutions to a semilinear Neumann problem. Comm. Pure Appl. Math. 44, 819-851 (1991) Zbl 0754.35042 MR 1115095

[12] Ni, W.-M., Takagi, I.: Locating the peaks of least energy solutions to a semilinear Neumann problem. Duke Math. J. 70, 247-281 (1993) Zbl 0796.35056 MR 1219814

[13] Ni, W.-M., Wei, J.: On the location and profile of spike-layer solutions to singularly perturbed semilinear Dirichlet problems. Comm. Pure Appl. Math. 68, 731-768 (1995) Zbl 0838.35009 MR 1342381

[14] Ni, W.-M., Wei, J.: Diffusion, cross-diffusion, and their spike-layer steady states. Notices Amer. Math. Soc. 45, 9-18 (1998) Zbl 0917.35047 MR 1490535 